\title{
Concept for an electrostatic focusing device for continuous ambient pressure aerosol concentration
}

\author{
Joseph L. Woo ${ }^{1, \mathrm{a}}$, Neha Sareen ${ }^{1, \mathrm{~b}}$, Allison N. Schwier ${ }^{1, \mathrm{c}}$, and V. Faye McNeill ${ }^{1}$ \\ ${ }^{1}$ Department of Chemical Engineering, Columbia University, New York, NY 10027, USA \\ ${ }^{a}$ currently at: Department of Chemical and Biomolecular Engineering, Lafayette College, Easton, PA 18042, USA \\ b currently at: US Environmental Protection Agency, New York, NY 10007, USA \\ ${ }^{c}$ currently at: US Department of State, Washington, DC 20520, USA
}

Correspondence: Joseph L.Woo (wooj@lafayette.edu) and V. Faye McNeill (vfm2103@columbia.edu)

Received: 5 February 2019 - Discussion started: 4 March 2019

Revised: 5 June 2019 - Accepted: 9 June 2019 - Published: 27 June 2019

\begin{abstract}
We present a concept for enhancing the concentration of charged submicron aerosol particles in a continuousflow stream using in situ electrostatic focusing. It is proposed that electrostatic focusing can enable the continuous, isothermal concentration of aerosol particles at ambient pressure, without altering their chemical composition. We model this approach theoretically and demonstrate proof of concept via laboratory measurements using a prototype. The prototype design consists of a nozzle-probe flow system analogous to a virtual impactor. The device was tested in the laboratory using submicron, monodisperse stearic acid particles. Particles were charged using a unipolar charger then concentrated using a cylindrical electrostatic immersion lens to direct the charged submicron particles into the sample probe. Under applied lens voltages ranging from $0 \mathrm{~V}$ to $30 \mathrm{kV}$, aerosol concentration increased up to $15 \%$. Observed particle enrichment varied as a function of voltage and particle diameter. These results suggest that an imposed electric field can be used to increase aerosol concentration in a continuous flow. This approach shows promise in increasing the effective enriched size range of virtual impactors or other continuousflow methods of collection.
\end{abstract}

\section{Introduction}

It is desirable for a number of applications to be able to continuously concentrate submicron aerosols at atmospheric pressure without affecting their chemical composition. For example, the leading techniques for detailed chem- ical characterization of the organic fraction of ambient particulate matter rely on particle collection-volatilization cycles (Williams et al., 2006; Lopez-Hilfiker, 2014). Other techniques that explore size-dependent properties of ambient (Mei et al., 2013; Thalman et al., 2017) or laboratorygenerated (Ahern et al., 2016; Petters et al., 2006; Vaden et al., 2011) organic aerosol using differential mobility analyzers (DMAs), such as Humidified Tandem DMA (HT-DMA) or cloud condensation nuclei counters (CCNCs), are complicated by the necessity of size selection of polydisperse samples, which work with a small mass fraction of a given aerosol sample's original population. Online (continuousflow) aerosol concentration would improve instrument signal and time response in these types of studies, simplifying data analysis.

Online aerosol concentration techniques typically involve inertial concentration. Approaches using aerodynamic lenses (Liu et al., 1995a, b) have been effective for aerosol mass spectrometry applications (Jayne et al., 2000), but the low ultimate pressure necessary for collection of 50-500 nm particles (Wang and McMurry, 2006) makes an aerodynamic lens inlet unsuitable for use with some detection techniques, such as chemical ionization mass spectrometry (CIMS) (Hearn and Smith, 2004; Sareen et al. 2010; Lopez-Hilfiker et al., 2014). Virtual impactor approaches (Chen et al., 1986) are effective for larger particles but less successful for concentrating submicron aerosols due to their low Stokes numbers (Seinfeld and Pandis, 2016). This has been addressed in the past via condensational growth of the particles prior to concentration (Kim et al., 2001a, b; Gupta et al., 2004; Geller 
et al., 2005; Saarikoski et al., 2019), though condensation onto aerosols can alter measured aerosol organic composition (Su et al., 2006; Jung et al., 2010). High aerosol flow velocities can also be used to achieve concentration of submicron particles but can lead to nonisothermal conditions, which are undesirable for analysis of semivolatile aerosols. Near-isothermal flow may be obtained by ensuring that the flow does not decelerate during sampling (Dhaniyala et al., 2003) or by restricting flow velocities to below the compressibility limit. Lee et al. (2003) presented and characterized a nanoparticle virtual impactor which used a convergentdivergent (de Laval) nozzle to achieve high flow velocities without shock waves (Lee et al., 2003), achieving a 50\% cutoff diameter of approximately $60 \mathrm{~nm}$ when operating at ambient pressure with an impactor chamber maintained at 220 Torr $(29.3 \mathrm{kPa})$. Thermal performance was not characterized in that study. Middha and Wexler (2006) built upon the Lee et al. (2003) inlet by designing a virtual impactor with a slot-shaped nozzle, which is the conceptual equivalent of several cylindrical nozzles functioning in parallel. This design was intended to produce high throughput and allow higher operating pressures than a single axisymmetric nozzle. Flow was nonisothermal in that device, with a predicted temperature gradient of $\sim 20^{\circ} \mathrm{C}$.

In this study, we present a concept for the application of in situ electrostatic focusing to isothermally concentrate a continuously flowing aerosol stream of submicron particles, at ambient pressure, without altering their chemical composition. Electrostatic precipitation has been used for decades to remove charged particles from gas streams and enhance particle collection for offline measurement techniques (Liu et al., 1967; Dixkens and Fissan, 1999; Fierz et al., 2007). We propose an approach based on a virtual impactor geometry (Chen et al., 1986), wherein an enriched aerosol stream can be continuously generated, such as those used in online characterization. A two-component cylindrical immersion lens is placed concentric to the gas-particle separation region (Fig. 1). The particles are charged upstream of this region and then passed through a radially symmetric, inwardfocusing electric field in order to focus them into a sampling probe. We model this approach theoretically and demonstrate proof of concept via laboratory measurements using a prototype. Homogeneously nucleated stearic acid is used as a representative organic aerosol for assessment of prototype performance.

\section{Design considerations}

As described in the previous section, in order to create an aerosol concentrator suitable for use upstream of AerosolCIMS or similar techniques, the following design requirements must be met: (1) concentration of submicron aerosol, (2) ultimate pressure of $\geq 2$ Torr $(0.266 \mathrm{kPa})$, (3) isothermal

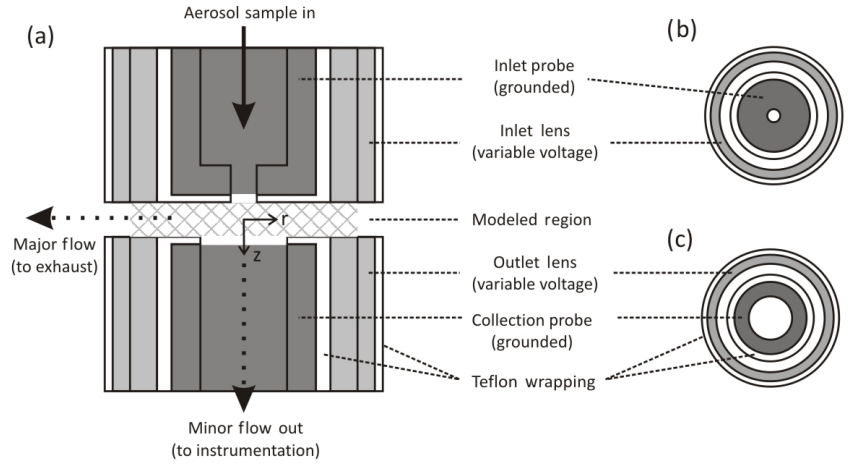

Figure 1. Schematic of the prototype electrostatic concentrator perpendicular to flow (a), down the inlet probe (b), and down the collection probe (c). Dark grey regions are grounded, white regions are PTFE insulation, and light grey regions are at variable voltage.

or near-isothermal sampling, and (4) no chemical alteration of particles from the sampling process.

Our approach is based on the concept that aerosol particles in a virtual impactor may deviate from the surrounding gas flow, depending on the balance of inertial, electrical, and viscous drag forces acting on the particle. For a charged particle in an electric field, the particle equation of motion is expressed as follows (Seinfeld and Pandis, 2016):

$m_{\mathrm{p}} \frac{\mathrm{d} \boldsymbol{v}}{\mathrm{d} t}=\frac{3 \pi \mu D_{\mathrm{p}}}{C_{\mathrm{c}}}(\boldsymbol{u}-\boldsymbol{v})+q \boldsymbol{E}$,

where $m_{\mathrm{p}}$ is the particle mass, $\boldsymbol{v}$ is the velocity vector of the particle, $\mu$ is the gas viscosity, $D_{\mathrm{p}}$ is particle diameter, $C_{\mathrm{c}}$ is the slip correction factor, $\boldsymbol{u}$ is the velocity vector of the carrier gas, $q$ is the charge on the particle, and $\boldsymbol{E}$ is the electric field vector. After non-dimensionalizing the above equation, we can derive a dimensionless parameter, $\kappa$, which describes the balances between electrostatic (deflective) and inertial forces. The derivation of $\kappa$ can be found in the Supplement.

$\kappa=\frac{Z \cdot E_{0}}{\mathrm{St} \cdot u_{0}}=\frac{6 L q E_{0}}{\rho \pi D_{\mathrm{p}}^{3} u_{0}^{2}}$

Here, $L$ is a characteristic length of the aerosol flow stream, set in this study as the gap distance between the orifice and collection probe defining our virtual impactor flow system. $Z$ is the electric mobility of the particle, and $\rho$ is the density of the aerosol particle. $E_{0}$ is a characteristic electric field strength. When $\kappa$ approaches or exceeds 1, electrostatic forces become sufficient to deflect submicron particles toward the sample probe. Note that for $\kappa \gg 1$ over-deflection and particle losses may occur. In Fig. 2, $\kappa$ is plotted as a function of electric field strength for a $30 \mathrm{~nm}$ particle flowing at a range of subsonic velocities. A variety of combinations of the variables satisfy $\kappa \geq 1$. 


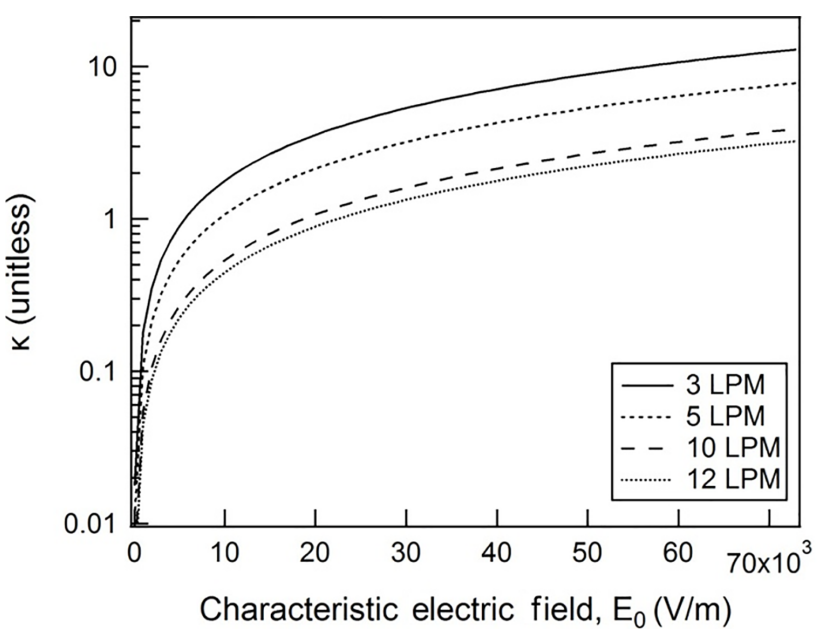

Figure 2. $\kappa$ versus voltage (V) for a range of velocities that satisfy the AC-CIMS design requirements, using prototype geometry. Particle diameter $30 \mathrm{~nm}$.

\subsection{Flow characterization}

Performance of the concentrator prototype was simulated in more detail using a numerical model that approximates the force balance on a particle in the proposed prototype geometry (see the following section for details). The trajectory of single particles through the concentrator was modeled, assuming gas flow characteristics of an inertial impactor with cylindrical geometry. Flow was approximated using potential flow of an incompressible gas into a stagnation point with dimensionless radial and axial coordinates, $\hat{r}$ and $\hat{z}$ (Bird et al., 2007):

$\widehat{u_{r}}=\hat{r}$

$\widehat{u_{z}}=\frac{1}{2}(1-\hat{z})$.

Here, $\hat{r}$ and $\hat{z}$ are dimensionless radial and axial positions scaled to the lens radius and half of the gap distance between the entrance orifice and collection probe, respectively. The origin of $\hat{r}$ and $\hat{z}$ was set to be the midpoint between the two cylindrical lenses, at the center of the axis of symmetry of the lenses (see Fig. 1). $\widehat{u_{r}}$ and $\widehat{u_{z}}$ are the dimensionless radial and axial velocities, both scaled to the initial entrance velocity. The initial trajectory of the gas-aerosol stream was defined within an orifice entrance region, described in further detail below, with an initial velocity of $\left(\widehat{u_{r}}, \widehat{u_{z}}\right)=(0,1)$.

\subsection{Electrical deflection}

We use a cylindrical immersion lens as a source of generating an electric field, though any geometry that is capable of generating radially inward electric fields while not interfering with bulk flow may be used. Immersion lenses are a commonly used method of manipulating streams of lowenergy electrons and particle beams (Páris, 1966; Heddle,
Table 1. Cylindrical lens functions $X_{0}, X_{1}$ (adapted from Bertram, 1942).

\begin{tabular}{lcc}
\hline & $1>\hat{z}>0$ & $-1<\hat{z}<0$ \\
\hline $\begin{array}{l}X_{0}(\hat{r}, \hat{z}) \\
(\text { Eq. 4a, b) }\end{array}$ & $1-\sum_{+\mu} \frac{J_{0}(\mu \hat{r})}{\mu J_{1}(\mu)} e^{-\mu \hat{z}}$ & $\sum_{+\mu} \frac{J_{0}(\mu \hat{r})}{\mu J_{1}(\mu)} e^{\mu \hat{z}}$ \\
\hline $\begin{array}{l}X_{1}(\hat{r}, \hat{z}) \\
(\text { Eq. 4c, d) }\end{array}$ & $\hat{z}+\sum_{+\mu} \frac{J_{0}(\mu \hat{r})}{\mu^{2} J_{1}(\mu)} e^{-\mu \hat{z}}$ & $\sum_{+\mu} \frac{J_{0}(\mu \hat{r})}{\mu^{2} J_{1}(\mu)} e^{\mu \hat{z}}$ \\
\hline
\end{tabular}

2000; Humphries, 1999; Read, 1971; Gillespie and Brown, 1997). By placing two charged conductive cylinders of equal radius in close proximity to one another, a radially symmetrical electric field is generated as a function of its geometry and the applied voltage gap between the two lenses.

A charged particle moving in the axial direction of the cylinders that enters this field is subject to two deflective forces: an axial accelerating force and a radial force that pushes both towards and away from the axis of symmetry. The net effect of these forces is a deflection of the overall path of the particle towards the center line and the entrance of the collection probe. A cylindrical lens assembly can be positioned over a virtual impactor geometry such that the submicron particles exiting the nozzle can be electrically deflected toward the collector (minor flow), without otherwise interfering with the gas flow field. The gap distance between the two component lenses was set to match that of the previously described virtual impactor gap distance.

The electric field generated from this assembly can be described as the gradient of the applied voltage field resulting from the immersion lens, which was approximated by Bertram (1942) for our geometry as

$$
\begin{aligned}
\hat{V} & =0.15\left(X_{0}(\hat{r}, \hat{z}+1)+X_{0}(\hat{r}, \hat{z}-1)\right) \\
& +0.7\left(X_{1}(\hat{r}, \hat{z}+1)+X_{1}(\hat{r}, \hat{z}-1)\right) .
\end{aligned}
$$

$X_{0}$ and $X_{1}$, seen in Table 1, are functions of the zeroth and first Bessel functions of the first kind, $J_{0}$ and $J_{1}$. In these equations, $\mu$ represents the set of positive roots of the zeroth order Bessel function, such that $J_{0}(\mu)=0$.

One thousand particle trajectories were modeled with and without the presence of applied voltage. The trajectories were initiated at the outlet of the orifice $(\hat{z}=-1)$, with radial positions spaced equally from $0<\hat{r}<0.1$ (see Fig. 1). A particle was considered to have been captured by the collection probe if its trajectory terminated within the collection region $(\hat{z}=1, \hat{r}<0.22)$.

The ratio of captured streams with and without applied electric fields, referred to as an enrichment ratio, was used to quantify the predicted performance of the prototype for a given set of conditions. Figure 3 shows the calculated enrichment factors for singly charged particles of typical aerosol density $\left(1 \mathrm{~g} \mathrm{~cm}^{-3}\right)$. 


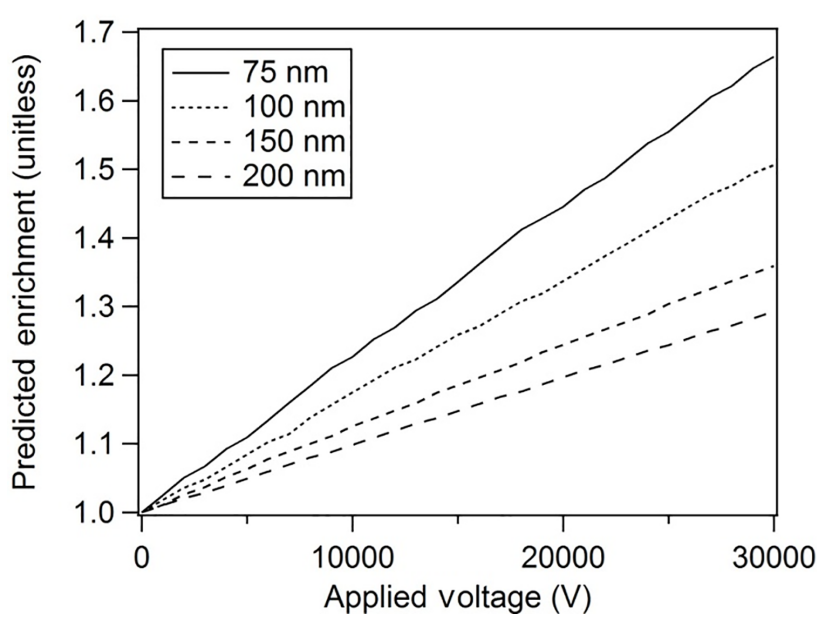

Figure 3. Predicted enrichment versus applied voltage under experimental operating conditions.

Table 2. Prototype concentrator dimensions.

\begin{tabular}{lr}
\hline Component & Length $(\mathrm{cm})$ \\
\hline Inlet probe orifice diameter & 0.1 \\
Inlet probe outer diameter & 0.635 \\
Inlet probe orifice length & 0.145 \\
Orifice-collection probe gap distance & 0.1 \\
Collection probe inner diameter & 0.444 \\
Collection probe outer diameter & 0.635 \\
Immersion lens inner diameter & 1.0 \\
Immersion lens outer diameter & 1.28 \\
Orifice approach angle & $90^{\circ}$ \\
\hline
\end{tabular}

\section{Prototype design}

A schematic of the relevant geometry of the prototype is shown in Fig. 1, with key dimensions listed in Table 2. Two stainless steel tubes of $0.444 \mathrm{~cm}$ inner diameter were attached into opposite ends of a KF-16 five-way cross vacuum fitting to act as an aerosol flow inlet and collection probe, respectively. A $0.1 \mathrm{~cm}$ inner diameter tube was bonded to the end of the inlet tube, forming a flow restriction. The ends of the inlet and collection probe were set $0.1 \mathrm{~cm}$ apart from one another, forming a gap for non-captured flow to travel through. The two junctions coplanar to the inlet and collection probe were connected to an exhaust, providing an exit for the major flow. All of these components were electrically connected to one another and grounded.

The lens assembly consists of two electrically isolated, $1.0 \mathrm{~cm}$ inner diameter, stainless steel tubes, which were mounted close to one another such that their ends matched the gap distance between the two flow tubes. A gastight plug fitted with two electrically isolated electrodes was installed on the top junction of the KF-16 five-way cross and wired to each of the two tubes. The lens assembly and the two flow probe tubes were wrapped in PTFE tape to eliminate potential electric field interactions in other parts of the concentrator setup. Voltage was applied to the two cylindrical lenses using a high voltage power source (Kepco). Under conditions without applied voltage, lenses were electrically grounded.

\section{Performance assessment}

The electrostatic focuser was tested using a monodisperse stream of stearic acid particles produced via homogeneous nucleation. The experimental setup for testing is shown in Fig. 4. Ultra-high purity nitrogen (TechAir) was introduced into a $1 / 4^{\prime \prime}$ ID glass tube filled with solid stearic acid (Fisher Scientific). This tube was wrapped with heating tape and brought to $120^{\circ} \mathrm{C}$ using a Variac (Staco Energy). The generated particles were then passed to a tube with a second heated region maintained at $120^{\circ} \mathrm{C}$. Moving the location of the secondary heated region allowed for fine control of the peak diameter of the generated stearic acid aerosol. The polydisperse aerosol stream was maintained at 1.5 LPM and sizeselected to a monodisperse aerosol stream (diameters ranging from 50 to $200 \mathrm{~nm}$ ) using a DMA (TSI Model 3080).

The monodisperse aerosol was then passed through a unipolar corona charger, producing a stream of positively charged particles. This stream was then diluted to a total flow of 15 LPM and introduced to the electrostatic focuser. The outflow of the collection probe tube was maintained at 1.5 LPM, with excess flow traveling to exhaust.

Particle concentrations were traced at $1 \mathrm{~s}$ resolution using a condensation particle counter (CPC; TSI Model 3775), with and without applied voltage. (An example of such a trace can be seen in Fig. 5.) Enrichment factors were evaluated by comparing the time-averaged total aerosol concentrations of the collection probe outflow. At each condition, particle size distributions were also checked for consistency, using a scanning mobility particle sizer (SMPS; GRIMM Inc).

The effect of the prototype concentrator on Aerosol-CIMS signal was also tested, using a custom-built mass spectrometer. Further description of this setup can be found in Sareen et al. (2010). After passing through the electrostatic concentrator, stearic acid aerosol was passed through a $23 \mathrm{~cm}$ long, $1.25 \mathrm{~cm}$ inner diameter stainless steel tube wrapped in heating tape, to volatilize aerosol input before entering the chemical ionization region. The external temperature of this volatilization region was maintained at $150^{\circ} \mathrm{C}$ using a thermocouple and temperature controller (Staco Energy). Gas-phase analyte molecules were detected as products of their interactions with $\mathrm{I}^{-}$reagent ions $(\mathrm{m} / z$ values of $284\left(\mathrm{C}_{18} \mathrm{H}_{35} \mathrm{O}_{2}^{-}\right), 319\left(\mathrm{C}_{18} \mathrm{H}_{35} \mathrm{O}_{2}^{-} \cdot 2 \mathrm{H}_{2} \mathrm{O}\right)$, and 409 $\left.\left(\mathrm{I}^{-} \cdot \mathrm{C}_{18} \mathrm{H}_{36} \mathrm{O}_{2}\right)\right)$ using a quadrupole mass spectrometer (Extrel).

Both the SMPS and Aerosol-CIMS used in this study sample at 1.5 LPM. All other flows of the electrostatic focuser were maintained using needle valves and monitored using a 


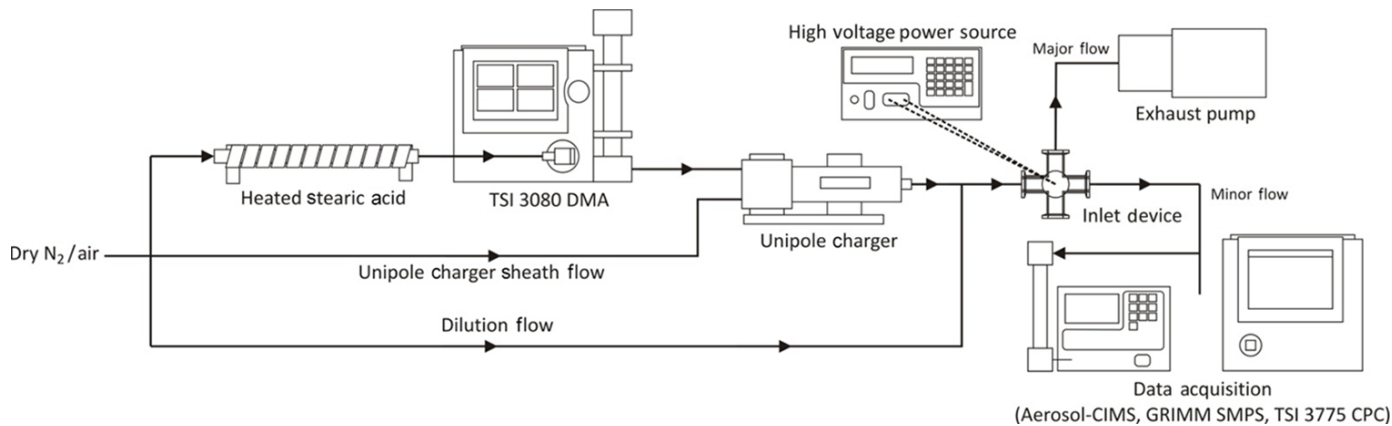

Figure 4. Experimental setup for evaluation of concentration gain from the electrostatic focuser.

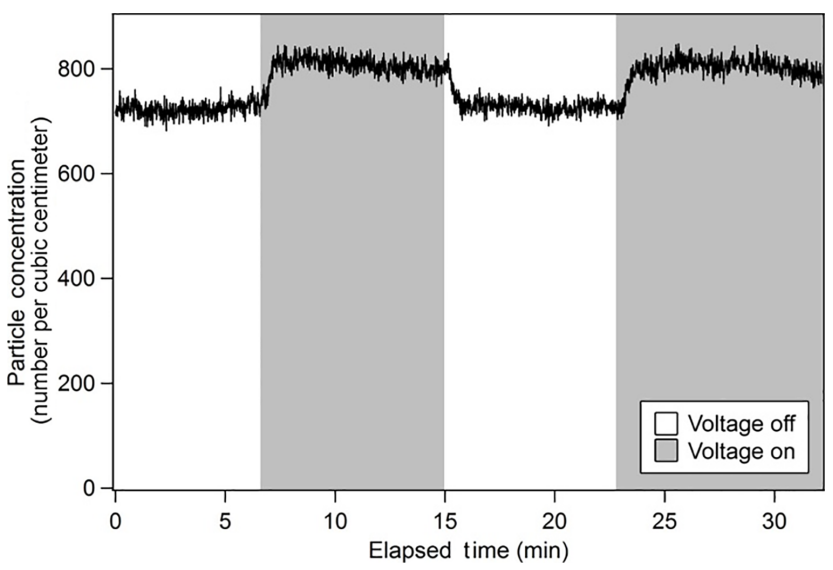

Figure 5. SMPS particle concentration trace with and without applied voltages. $200 \mathrm{~nm}, 30 \mathrm{kV}$ applied voltage.

mass flow meter (MKS Instruments). The major (exhaust) flow was controlled using a throttled mechanical pump (Varian DS302) and monitored with a separate, volumetric gas flow meter.

\section{Results and discussion}

When voltage is applied to the outlet lens (viz. Fig. 1), an increase in the particle concentration of the minor flow stream is observed (Fig. 5). The observed enrichment is summarized in Fig. 6, across a range of five aerosol diameters and three levels of applied voltage. As shown in Fig. 6, at $30 \mathrm{kV}$ applied voltage the dependence of the observed enrichment on particle size is opposite that predicted due to the model. This may be a result of over-deflection of the particles with the highest mobilities (i.e., the smallest particles) via the imposed electric field. No discernible change in sampled particle concentration was observed when voltage was applied to the inlet lens. Subsequent references to applied voltage in this discussion will refer to that applied to the outlet lens only.

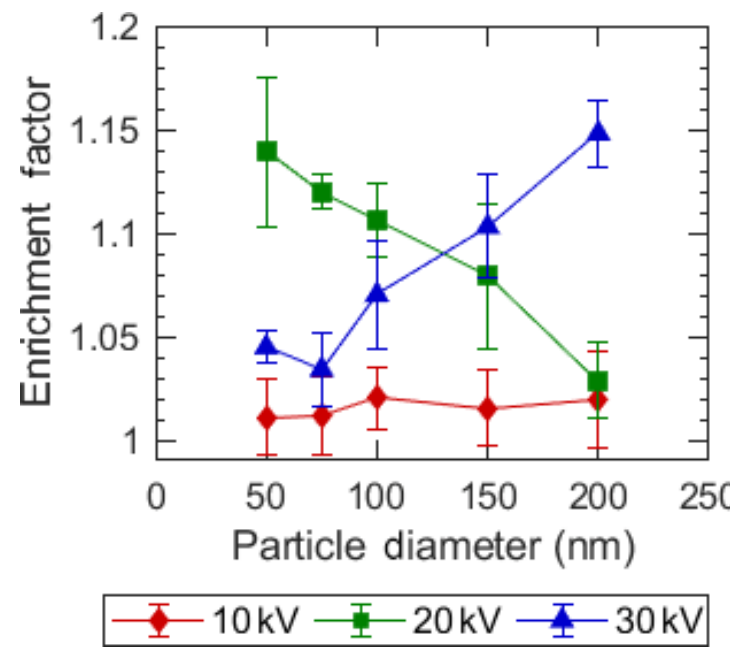

Figure 6. Observed aerosol enrichment at varying voltages and particle diameters.

We note that, while its design was based on virtual impactor geometry, at the pressure and flow conditions used here, the concentrator prototype does not operate as a true virtual impactor for submicron particle sizes. That is, with no applied voltage there is no inertial concentration of submicron particles, and the particle concentrations in the inlet, major stream, and minor stream were identical within the noise. Hence, the results shown here successfully demonstrate the isolated effect of electrostatic aerosol concentration.

Aerosol-CIMS traces in the absence and presence of voltage indicated enrichment consistent with the mass enrichment that was observed via the SMPS. No additional signal peaks beyond those of stearic acid were observed in AerosolCIMS during operation regardless of applied voltage, implying that stearic acid did not undergo any chemical reactions as a result of the charging process or from exposure to the electric field of the electrostatic focuser. Corona dischargebased unipolar chargers, such as the one employed in this study, are known to generate ozone at concentrations as high as 5 ppm near the filament (Wang and McMurry, 2006). Un- 
like stearic acid, unsaturated organic species such as oleic acid could therefore be vulnerable to $\mathrm{O}_{3}$ oxidation within the device. The expected residence time inside the unipolar charger used in this study is estimated at less than $10 \mathrm{~s}$, and the charger outflow is diluted by a factor of 10 , reducing the potential impact of oxidation. However, the risk of unwanted chemical oxidation could be eliminated by using an alternative method of imposing charges onto the input aerosol stream that does not generate ozone (e.g., Han et al., 2008).

Calculated values for the Mach number reach as high as 0.94 within the entrance orifice, beyond the generally accepted threshold of incompressible flow (Kundu, 1990). However, due to the relatively short length scale of the entrance orifice, the predicted ratio of pressures across the inlet entrance is expected to be sufficiently low $(\sim 1.05)$ to avoid choked flow (Miller, 1996). Furthermore, temperature at various locations within the prototype was measured using a thermocouple after extended operation, and no significant temperature fluctuations were observed. Finally, AerosolCIMS traces for stearic acid in the absence of volatilization did not show any deviation from background values as a function of the flow velocity through the concentrator, consistent with this conclusion.

At all voltages tested, observed enrichment was lower than model predicted values under the same conditions. The aerosol enrichment observed via SMPS and Aerosol-CIMS corresponded to that predicted for voltages that were between $15 \%$ and $20 \%$ of those actually applied. We believe that this discrepancy between the model and observations is largely due to distortion of the electric fields within the gas-particle separation region. Additional minor losses could be due to particle losses inside the sample probe.

As the deflection of aerosol within the inlet system is highly dependent on the charged particle optics within the immersion lens geometry, even slight physical deviations of the relative orientation of the metal components comprising the lens will lead to distortions in their resultant electric fields when voltages are applied. Of note, translational and angular asymmetries that arise in misalignment of the two lens elements will lead to spherical field aberrations (astigmatism, coma, etc.) (Heddle, 2000). These aberrations introduce radial and angular electric field asymmetries, which may result in uneven or misdirected deflection of aerosols as they pass through the lens. Physical imperfections in the lens elements themselves (i.e., machine imperfections, surface roughness, etc.) will also introduce further deviations from idealized immersion lens electric fields, resulting in reduced enrichment compared to theoretical values estimated in Fig. 3.

Electric field distortion is also possible from other solid surfaces within the lens assembly, especially the collection probe, due to its close proximity to the outlet lens. As previously mentioned, the collection probe was covered with PTFE, but this likely provided incomplete electrical insulation. In order to evaluate the potential impact of field distortion on the performance of the electrostatic focuser, we estimated the magnitude of the electric field generated by the potential between the outlet lens and the collection probe in a worst-case scenario in the absence of any insulation (see the Supplement for details of the calculation). We approximated the fringe effects of the two concentric cylinders (the outlet lens and the collection probe) as being similar to that derived from two parallel plates. Several studies have examined the effective field strength, voltage distribution, and charge densities of the edges of finite plates of infinite width (Pillai, 1970; Parker, 2002). The two relevant voltage distributions, described by Bertram (1942; inlet lens-outlet lens) and Parker (2002; outlet lens-collection probe), were combined in order to estimate their relative effects on the total electric field. In the worst-case scenario of an uninsulated collection probe, the magnitude of the radial component of the combined electric field becomes negligible within the outer radius of the collection probe. That is, electrostatic enrichment of the aerosol becomes negligible. These calculations assume that the collection probe is exposed and conductive. Taking measures to decrease its behavior as a source of undesired electric fields, such as the PTFE tape insulation employed in this study, will help control distortion and lead to enrichments closer to theoretical values.

Fierz et al. (2007) used electrostatic focusing to enhance particle collection via inertial impaction onto a transmission electron microscopy (TEM) grid in a radially symmetric design, reminiscent of the one employed here. They achieved $7 \%-9 \%$ collection efficiency for $50 \mathrm{~nm}$ aerosol at an applied voltage of $3 \mathrm{kV}$, which is close to our theoretical enrichment $(12 \%)$ at the same voltage. We expect that the electric field in their system, which was generated via the potential between a lens and their collection plate, was relatively free of distortion, due to the absence of additional nearby solid surfaces in the gas-particle separation region (which would not be possible in a continuous-flow design such ours). This provides further support for the notion that distortion of the electric field was the primary cause of the observed discrepancy between the model predictions and observed enrichment.

It should be restated that the cylindrical lens geometry in the design used here was implemented because of its relatively simple construction and lack of interference with the gas flow field. For the desired focusing effect, a net radially inward component of the electric field is needed, but the exact geometry of the electric fields and the elements needed to generate them are nonspecific. As our experimental results have indicated, so long as a sufficiently strong electrical deflective force can be placed opposite to an inertial flow force in the radial direction, modulation and concentration of an aerosol stream can be achieved.

Pressures inside the aerosol concentrator were kept at ambient or positive values in this study, but our model outputs suggest that this geometry can be implemented at lower pressures without affecting the net deflection forces of an imposed electric field. However, lower gas pressures inside the charging region can decrease the effective breakdown voltage 
between the two cylindrical lenses of this system, increasing the probability of arcing.

\section{Summary and conclusions}

We have demonstrated a novel concept for enhancing the concentration of submicron charged aerosol particles in a gas stream by means of electrostatic focusing. Stearic acid aerosol concentration enrichments up to $15 \%$ were observed using a proof-of-concept prototype under applied voltages up to $30 \mathrm{kV}$. This approach is compatible with analytical methods that require isothermal, ambient pressure sampling and relatively high ultimate pressures. Our prototype employed a cylindrical immersion lens and a virtual impactor geometry. Although flow velocities used here were too low for concentration of submicron aerosol aerodynamically using virtual impaction, electrostatic focusing could enhance the performance of a virtual impactor, allowing the concentration of smaller particles. We note that, besides a cylindrical immersion lens, electrostatic focusing of particles may be also achieved by a charged mesh tube, cylindrical lens assembly (e.g., Einzel lens), octupole, or other means of generating a radially inward-facing electric field.

Data availability. The data and models used to generate the figures presented in this work can be made available for research purposes via email request to the corresponding authors (Joseph L. Woo and V. Faye McNeill.)

Supplement. The supplement related to this article is available online at: https://doi.org/10.5194/amt-12-3395-2019-supplement.

Author contributions. VFM developed the original concept for this work. JLW, VFM, and NS performed derivations of $\kappa$ and theoretical enrichment values. JLW implemented the MATLAB code simulations to evaluate theoretical enrichment values and constructed the immersion lens system evaluated in this work. JLW, NS, and ANS carried out the experiments necessary to measure observed enrichment. VFM and JLW wrote the paper.

Competing interests. The authors declare that they have no conflict of interest.

Acknowledgements. The authors acknowledge the NSF BRIGE program (EEC-0823847) for funding. The authors thank Coty Jen for valuable discussion and technical assistance in early stages of this work.
Financial support. This research has been supported by the National Science Foundation, Division of Chemical, Bioengineering, Environmental, and Transport Systems (grant no. EEC-0823847).

Review statement. This paper was edited by Mingjin Tang and reviewed by three anonymous referees.

\section{References}

Ahern, A. T., Subramanian, R., Saliba, G., Lipsky, E. M., Donahue, N. M., and Sullivan, R. C.: Effect of secondary organic aerosol coating thickness on the real-time detection and characterization of biomass-burning soot by two particle mass spectrometers, Atmos. Meas. Tech., 9, 6117-6137, https://doi.org/10.5194/amt-96117-2016, 2016.

Bertram, S.: Calculation of axially symmetric fields, J. Appl. Phys., 13, 496-502, 1942.

Bird, R. B., Stewart, W. E., and Lightfoot, E. N.: Transport Phenomena, 2nd edn., John Wiley \& Sons, New York, 2007.

Chen, B. T., Yeh, H. C., and Cheng, Y. S.: Performance of a Modified Virtual Impactor, Aerosol Sci. Technol., 5, 369-376, 1986.

Dhaniyala, S., Flagan, R. C., McKinney, K. A., and Wennberg, P. O.: Novel aerosol/gas inlet for aircraft-based measurements, Aerosol Sci. Technol., 37, 828-840, 2003.

Dixkens, J. and Fissan, H: Development of an Electrostatic Precipitator for Off-Line Particle Analysis, Aerosol Sci. Technol., 30, 438-453, 1999.

Fierz, M., Kaegi, R., and Burtscher, H.: Theroetical and Experimental Evaluation of a Portable Electrostatic TEM Sampler, Aerosol Sci. Technol., 41, 520-528, 2007.

Geller, M. D., Biswas, S., Fine, P. M., and Sioutas, C.: A new compact aerosol concentrator for use in conjunction with low flowrate continuous aerosol instrumentation, Aerosol Sci., 36, 10061022, 2005.

Gillespie, G. H. and Brown, T. A.: Optics elements for modeling electrostatic lenses and accelerator components I. Einzel lenses, in: Proceedings of the 1997 Particle Accelerator Conference, 1216, Vancouver, vol.2552, 2559-2561, 1997.

Gupta, T., Demokritou, P., and Koutrakis, P.: Development and Performance Evaluation of a High-Volume Ultrafine Particle Concentrator for Inhalation Toxicological Studies, Inhalation Toxicol., 16, 851-862, 2004.

Han, B., Kim, H.-J., Kim, Y.-J., and Sioutas, C: Unipolar Charging of Fine and Ultra-Fine Particles Using Carbon Fiber Ionizers, Aerosol Sci. Technol., 42, 793-800, 2008.

Hearn, J. D. and Smith, G. D.: A chemical ionization mass spectrometry method for the online analysis of organic aerosols, Anal. Chem., 76, 2820-2826, 2004.

Heddle, D. W. O.: Electrostatic Lens Systems, Institute of Physics Publishing, London, 2000.

Humphries, S.: Principles of Charged Particle Acceleration, John Wiley \& Sons, Albuquerque, New Mexico, 1999.

Jayne, J. T., Leard, D. C., Zhang, X., Davidovits, P., Smith, K. A., Kolb, C. E., and Worsnop, D. R.: Development of an Aerosol Mass Spectrometer for Size and Composition Analysis of Submicron Particles, Aerosol Sci. Technol., 33, 49-70, 2000. 
Jung, H., Arellanes, C., Zhao, Y., Paulson, S., Anastasio, C., and Wexler, A.: Impact of the Versatile Aerosol Concentration Enrichment System (VACES) on Gas Phase Species, Aerosol Sci. Technol., 44, 1113-1121, 2010.

Kim, S., Jaques, P. A., Chang, M., Froines, J. R., and Sioutas, C.: Versatile aerosol concentration enrichment system (VACES) for simultaneious in vivo and in vitro evaluation of toxic effects of ultrafine, fine, and coarse ambient particles, Part I: Development and laboratory characterization, J. Aerosol Sci., 32, 1281-1297, 2001a.

Kim, S., Jaques, P. A., Chang, M., Barone, T., Xiong, C., Friedlander, S. K., and Sioutas, C.: Versatile aerosol concentration enrichment system (VACES) for simultaneous in vivo and in vitro evaluation of toxic effects of ultrafine, fine and coarse ambient particles Part II: Field evaluation, Aerosol Sci., 32, 1299-1314, 2001b.

Kundu, P. K.: Fluid Mechanics, Academic Press, Inc., San Diego, CA, 1990.

Lee, P., Chen, D.-R., and Pui, D. Y. H.: Experimental study of a nanoparticle virtual impactor, J. Nanoparticle Res., 5, 269-280, 2003.

Liu, B. Y., Whitby, K. T., and Yu, H. H. S.: Electrostatic Aerosol Sampler for Light and Electron Microscopy, Rev. Sci. Instru., 38, 100-102, 1967.

Liu, P., Ziemann, P. J., Kittelson, D. B., and McMurry, P. H.: Generating Particle Beams of Controlled Dimensions and Divergence, 1. Theory of Particle Motion in Aerodynamic Lenses and Nozzle Expansions, Aerosol Sci. Technol., 22, 293-313, 1995a.

Liu, P., Ziemann, P. J., Kittelson, D. B., and McMurry, P. H.: Generating Particle Beams of Controlled Dimensions and Divergence, 2. Experimental Evaluation of Particle Motion in Aerodynamic Lenses and Nozzle Expansions, Aerosol Sci. Technol., 22, 314324, $1995 \mathrm{~b}$.

Lopez-Hilfiker, F. D., Mohr, C., Ehn, M., Rubach, F., Kleist, E., Wildt, J., Mentel, Th. F., Lutz, A., Hallquist, M., Worsnop, D., and Thornton, J. A.: A novel method for online analysis of gas and particle composition: description and evaluation of a Filter Inlet for Gases and AEROsols (FIGAERO), Atmos. Meas. Tech., 7, 983-1001, https://doi.org/10.5194/amt-7-983-2014, 2014.

Mei, F., Setyan, A., Zhang, Q., and Wang, J.: CCN activity of organic aerosols observed downwind of urban emissions during CARES, Atmos. Chem. Phys., 13, 12155-12169, https://doi.org/10.5194/acp-13-12155-2013, 2013.

Middha, P. and Wexler, A. S.: Design of a slot nanoparticle virtual impactor, Aerosol Sci. Technol., 40, 737-743, 2006.

Miller, R. W.: Flow Measurement Engineering Handbook, 3rd edn., McGraw-Hill Professional, New York, 1996.

Páris, G. Y.: Approximations to immersion type electrostatic lenses, Nucl. Inst. Meth., 44, 137-140, 1966.

Parker, G. W.: Electric field outside a parallel plate capacitor, Am. J. Phys., 70, 502-507, 2002.
Petters, M. D., Kreidenweis, S. M., Snider, J. R., Koehler, K. A., Wang, Q., Prenni, A. J., and Demott, P. J.: Cloud droplet activation of polymerized organic aerosol, Tellus B, 58, 196-205, https://doi.org/10.1111/j.1600-0889.2006.00181.x, 2006.

Pillai, K. P. P.: Fringing field of finite parallel plate capacitors, Proc. IEE, 117, 1201-1204, 1970.

Read, F. H.: Electrostatic cylinder lenses. I. Two element lenses, J. Phys. E, 4, 625-632, https://doi.org/10.1088/0022-3735/4/9/001, 1971.

Saarikoski, S., Williams, L. R., Spielman, S. R., Lewis, G. S., Eiguren-Fernandez, A., Aurela, M., Hering, S. V., Teinilä, K., Croteau, P., Jayne, J. T., Hohaus, T., Worsnop, D. R., and Timonen, H.: Laboratory and field evaluation of the Aerosol Dynamics Inc. concentrator (ADIc) for aerosol mass spectrometry, Atmos. Meas. Tech. Discuss., https://doi.org/10.5194/amt-201974, in review, 2019.

Sareen, N., Schwier, A. N., Shapiro, E. L., Mitroo, D., and McNeill, V. F.: Secondary organic material formed by methylglyoxal in aqueous aerosol mimics, Atmos. Chem. Phys., 10, 997-1016, https://doi.org/10.5194/acp-10-997-2010, 2010.

Seinfeld, J. H. and Pandis, S. N.: Atmospheric chemistry and physics: From air pollution to climate change, 3rd edn., Wiley, New York, 2016.

Su, Y., Sipin, M. F., Spencer, M. T., Qin, X., Moffet, R. C., Shields, L. G., Prather, K. A., Venkatachari, P., Jeong, C.-H., Kim, E., Hopke, P. K., Gelein, R. M., Utell, M. K., Oberdorster, G., Bernsten, J., Devlin, R. B., and Chen, L. C.: Real-time Characterization of the Composition of Individual Particles Emitted from Ultrafine Particle Concentrators, Aerosol Sci. Technol., 40, 437455, 2006.

Thalman, R., de Sá, S. S., Palm, B. B., Barbosa, H. M. J., Pöhlker, M. L., Alexander, M. L., Brito, J., Carbone, S., Castillo, P., Day, D. A., Kuang, C., Manzi, A., Ng, N. L., Sedlacek III, A. J., Souza, R., Springston, S., Watson, T., Pöhlker, C., Pöschl, U., Andreae, M. O., Artaxo, P., Jimenez, J. L., Martin, S. T., and Wang, J.: CCN activity and organic hygroscopicity of aerosols downwind of an urban region in central Amazonia: seasonal and diel variations and impact of anthropogenic emissions, Atmos. Chem. Phys., 17, 11779-11801, https://doi.org/10.5194/acp-1711779-2017, 2017.

Vaden, T. D., Imre, D., Beránek, J., Shrivastava, M., and Zelenyuk, A.: Evaporation kinetics and phase of laboratory and ambient secondary organic aerosol, P. Natl. Acad. Sci. USA, 108, 21902195, https://doi.org/10.1073/pnas.1013391108, 2011.

Wang, X. L. and McMurry, P. H.: A design tool for aerodynamic lens systems, Aerosol Sci. Technol., 40, 320-334, 2006.

Williams, B. J., Goldstein, A. H., Kreisberg, N. M., and Hering, S. V.: An in-situ instrument for speciated organic composition of atmospheric aerosols: Thermal Desorption Aerosol GC/MS-FID (TAG), Aerosol Sci. Technol., 40, 627-638, 2006. 